\title{
Preventive Potential Of Borneol In Ischemic Stroke
}

Hadi Esmaeeli, Ramin Ataei

Borneol is a bicyclic organic compound found in several species of Artemisia, Dipterocarpaceae and etc. It can easily permeate through the blood brain barrier and acts directly in neurons. Ischemic stroke, an imbalance of the blood supply to the brain because of blood clots, is a severe condition with high mortality and morbidity that represents $87 \%$ of all stroke cases. The aim of this study is to evaluate the effect of borneol in the prevention of stroke.

Borneol can be synthesized by reduction of camphor by the Meerwein-Ponndorf-Verley reduction. Protection against Oxygen-Glucose Deprivation/Reperfusion Injury in Cortical Neurons by antioxidation and anti-inflammation through nuclear transcription factor карраB signaling pathway is one of suggested mechanisms for borneol. Combination of some compounds and borneol can have a synergistic effect on treating ischemic stroke in a rat model. Also borneol permeating can increased into brain in the pathological state of stroke. This review was made to help better understanding of the borneol evaluation in ischemic stroke prevention.

Key words: Borneol, Ischemic stroke, Blood brain barrier 